\title{
Fast SCOP Classification of Structural Class and Fold Using Secondary Structure Mining in Distance Matrix
}

\author{
Jian-Yu Shi ${ }^{1,2}$ and Yan-Ning Zhang ${ }^{2}$ \\ ${ }^{1}$ Faculty of Life Sciences, Northwestern Polytechnical University \\ ${ }^{2}$ College of Computer Science, Northwestern Polytechnical University \\ 710072 Xi'An, China \\ \{JianyuShi, YnZhang $\}$ @nwpu . edu.cn
}

\begin{abstract}
It is an urgent need to understand the structure-function relationship in proteomic era. One of the important techniques to meet this demand is to analyze and represent the spatial structure of domain which is the functional unit of the whole protein, and perform fast domain classification. In this paper, we introduce a novel method of rapid domain classification. Instead of analyzing directly protein sequence or 3-D tertiary structure, the presented method maps firstly tertiary structure of protein domain into 2-D C $\alpha-\mathrm{C} \alpha$ distance matrix. Then, two distance functions for alpha helix and beta strand are modeled by considering their geometrical properties respectively. After that, the distance functions are further applied to mine secondary structure elements in such distance matrix with the way similar to image processing. Furthermore, composition feature and arrangement feature of secondary structure elements are presented to characterize domain structure for classification of structural class and fold in Structural Classification of Proteins (SCOP) database. Finally, the results compared with other methods show that the presented method can perform effectively and efficiently automatic classification of domain with the benefit of low dimension and meaningful features, but also no need of complicated classifier system.
\end{abstract}

Keywords: SCOP classification, protein structure, distance matrix, secondary structure mining, image processing, support vector machines.

\section{Introduction}

The function of protein is strongly related to its spatial structure[1]. In order to understand the relationship of structure-function and discover the evolutional explanation of conserved structure, biologists and researchers have the need to retrieve similar tertiary structures for protein structure database, and further categorize them into different classes in terms of their secondary structure, topology and evolution information. Now, the representation, classification and retrieving of protein spatial structure has become the popular area in computational biology and structural bioinformatics.

However, the number of proteins with determined spatial structures but unknown types and unclear functions is still large and increasing continuously. Besides, current 
structural classification databases are constructed manually by numerous biologists[2], or implemented automatically by exhausted and inaccurate computation[3]. Along with the determination of more and more protein spatial structures, biologists raise the requirement of the classification of protein structure with not only the automatic way but also more accurate and lower computation cost. Consequently, how to represent the spatial structure of protein and perform fast structural classification has become the urgent need.

Based on the widely assumption that structural features are closely related to sequence composition[1, 4], one popular approach is called indirect representation of protein spatial structure which extracts feature of sequence to perform classification. The indirect representation can be organized into two types: based on statistical analysis of amino acid residues[5-8], and based on amino acid indices[9,10]. Another approach executes directly analysis on protein spatial structure to obtain representation and extract feature of structure, and can be grouped into three types: based on spatial atom distribution[11,12], topological structure[13,14], and geometrical shape[15-17]. The indirect representation can be always obtained with lower computation cost and higher dimensional feature, but in contrast the direct representation can be acquired with higher computation cost and lower dimensional feature. Besides, in order to achieve better classification with the indirect representation of protein structure, there is always a need to exploit advance techniques of pattern recognition, for example, feature combination[5], fusion[18,19], selection[7] and hierarchical classifier architecture[20,21].

In this paper, we present a feature extraction of protein spatial structure to achieve fast SCOP classification at structural class and fold levels. This paper is constructed as follows: Section 2 describes the benchmark dataset of protein structure used in this paper. Section 3 depicts the core of our idea about how to characterize protein structure in 2D matrix and represent it with a compact feature vector. Section 4 presents experimental results and investigates the effectiveness of our proposed algorithm. Finally, we draw the conclusions in section 5.

\section{Database}

Structural domains of protein often form functional units of which each forms a compact three-dimensional structure and often can be independently stable and folded. Protein always consists of one or more structural domains. On the other hand, one domain may appear in a variety of evolutionarily related proteins. Therefore, one of the important techniques to understand the structure-function relationship is the analysis of the spatial structure of protein domain.

Structural Classification of Proteins (SCOP) database is of a manual classification of protein structural domains based on similarities of their amino acid sequences and three-dimensional structures[2]. Because SCOP classification based on the human expertise is more significantly than semi-automatic CATH[3], it is usually accepted that SCOP provides a better justified classification. SCOP utilizes four levels of hierarchical classification: structural class, fold, superfamily and family. The classifications of 
structural class and fold are concerned about structural similarity, while the classifications of superfamily and family are devoted to sequence similarity.

The SCOP domain dataset used here is derived from the work[5] with high cited number, and can be downloaded from http://ranger.uta.edu/ chqding/protein/ or SCOP database[22]. It contains a training set and a testing set of which the sample counts are 313 and 385, the sequence similarities are less 35\% and $40 \%$ respectively. According to SCOP classification, the whole dataset consists of 4 kinds of structural classes which can be further categorized into 27 types of folds. Only the first two levels of SCOP classification are considered for the analysis of domain structure in this paper.

\section{Method}

\subsection{Distance Matrix}

Various physical and chemical properties, different counts and sequenced order of amino acids are the elements to decide and product the diversity of protein structures. As a result, it is complicated to describe directly protein structure by its all atoms, and even more difficult to analyze and characterize its structure for further understanding structure-function relationship. Instead of considering all atoms, many computational biology literatures therefore use $C_{\alpha}$ atoms of protein to characterize the whole protein structure in order to obtain lower complexity of computation. It is also known as protein backbone[23]. As a kind of protein structure representations based on backbone, distance matrix (DM) contains sufficient information to reconstruct the original 3D backbone structure by using the distance geometry methods[24].

Suppose protein $P_{i}$ is composed of $\mathrm{N}$ amino acid residues and its backbone can be defined as $B_{i}=\left\{\operatorname{Coor}_{1}^{i}, \operatorname{Coor}_{2}^{i}, \cdots, \operatorname{Coor}_{n}^{i}, \cdots, \operatorname{Coor}_{N}^{i}\right\}$ where $\operatorname{Coor}_{n}^{i}$ is the coordinates vector of the $\mathrm{n}^{\text {th }} \mathrm{C} \alpha$ atom. Then, $B_{i}$ can product distance matrix

$$
D M=\left\{d m_{i}(p, q)=\operatorname{dist}\left(\operatorname{Coor}_{p}^{i}, \operatorname{Coor}_{q}^{i}\right)\right\}
$$

where $\operatorname{dist}(\cdot)$ is just the Euclidean distance between the $\mathrm{p}^{\text {th }}$ and the $\mathrm{q}^{\text {th }} \mathrm{C} \alpha$ atoms, and $1 \leq p, q \leq N$.

Since DM maintains sufficient 3-D structural information, similar protein backbones are expected to have such distance matrices with similar properties. Fig. 1 gives the structure snapshots and the images of DMs of four proteins which fall into all $\alpha$, all $\beta, \alpha / \beta$ and $\alpha+\beta$ structural class and four kinds of folds respectively.

As shown in Fig. 1, different kinds of protein structures have distinct DMs, that is to say, the difference of structures can be exhibited by their DMs. More importantly, secondary structure elements (SSEs) show regular patterns in DM. 


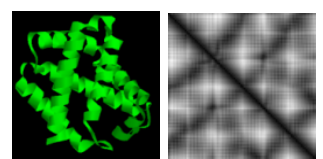

(a) d1hbga_, all $\alpha$, Globin-like, chain length 147
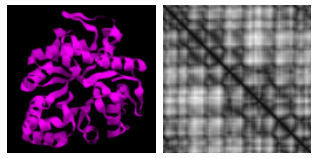

(c) d1ghra_, $\alpha / \beta$, TIM-barrel, chain length 306

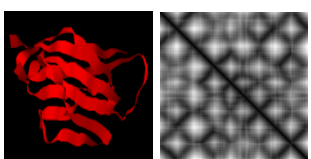

(b) d1neua_, all $\beta$, Immunoglobulin-like, chain length 115
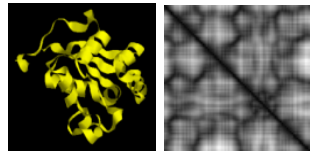

(d) d1npka_, $\alpha+\beta$, Ferredozin-like, chain length 150

Fig. 1. Structure snapshots and distance matrix images of four proteins

\subsection{Secondary Structure Mining}

The definitions of structural class and fold inspired us that the analysis of secondary structures composition and arrangement based on DM can hopefully represent protein structure with compact and effective way. However, SCOP domain file provides no information of secondary structure, there is a principal need to mine secondary structure in order to extract structural feature of protein domain.

There are two of the most popular SSEs: alpha-helix and beta-strand. Geometrically, the backbone of alpha-helix is a kind of spring, while the backbone of betastrand is a stretch of periodic pleating and two strands are connected laterally by three or more hydrogen bonds to forming pleated beta sheet.

In detail, the $\mathrm{C} \alpha$ atoms in an alpha-helix are arranged in a right-handed helical structure, $5.4 \AA$ wide, and each $\mathrm{C} \alpha$ atom corresponds to a $100^{\circ}$ turn in the helix, radius of $2.3 \AA$ and a translation of $1.5 \AA$ along the helical axis. Therefore, the distance between $\mathrm{C} \alpha(\mathrm{i})$ and $\mathrm{C} \alpha(\mathrm{i}+\mathrm{t})$ atoms in alpha-helix can be determined by

$$
l_{i \rightarrow i+t}^{\alpha}=\sqrt{r^{2}(1-2 \cos (t \cdot \theta))+(t \cdot d)^{2}}, t=1, \ldots,
$$

where $\mathrm{r}=2.3 \AA, \mathrm{d}=1.5 \AA$ and $\theta=100^{\circ}$.

Due to tetrahedral chemical bonding at the $\mathrm{C} \alpha$ atom, the pleated appearance of beta-strand causes the distance between $\mathrm{C} \alpha(\mathrm{i})$ and $\mathrm{C} \alpha(\mathrm{i}+2)$ to be approximately $6 \AA$, rather than the $\operatorname{sum}(2 \times 3.8 \AA)$ of the distances of adjacent $\mathrm{C} \alpha$ atom pairs. Therefore, the distance between $\mathrm{C} \alpha(\mathrm{i})$ and $\mathrm{C} \alpha(\mathrm{i}+\mathrm{t})$ atoms in beta-strand can be determined by

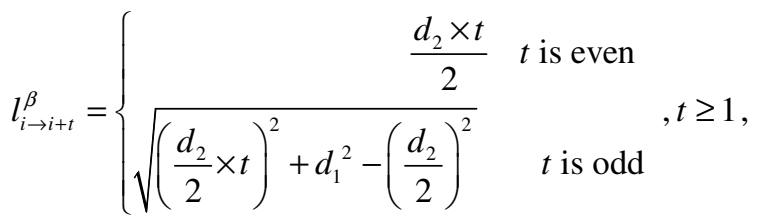

where $\mathrm{d}_{1}=3.8 \AA$, and $\mathrm{d}_{2}=6 \AA$. 
Because of symmetry of distance matrix, its upper triangular part is enough to mine SSEs by formula (2) or (3). However, there is the divergence of the distance of adjacent $\mathrm{C} \alpha-\mathrm{C} \alpha$ atoms in different protein derived from different experiments. As a result, we cannot apply these two formulas directly to find which residue is a participant of alpha helix or beta strand.

Here, inspired by the techniques of image processing, we determinate whether a residue belongs to a SSE or not by whether the following formula holds or not

$$
S_{i}=\sqrt{\sum_{k=1}^{m}\left(R_{k}^{i}-\bar{R}_{m}^{i}\right)^{2}}-\tau \leq 0,
$$

where $\tau$ is a threshold which controls the fitting error between distance function and elements of DM,

$$
\bar{R}_{m}^{i}=\sum_{k=1}^{m} R_{k}^{i} / m, R_{k}^{i}=\left\{\frac{\Delta D(i, k)}{\Delta L(i, k)}\right\}, i=1, . . k=1, \ldots,
$$

$\Delta D(i, k)=d m(i, i+k)-d m(i, i+k-1)$ and $\Delta L(i, k)=\left(l_{i \rightarrow i+k}-l_{i \rightarrow i+k-1}\right)$.

By considering the fact that the $\mathrm{N}-\mathrm{H}$ group of an amino acid forms a hydrogen bond with the $\mathrm{C}=\mathrm{O}$ group of the amino acid four residues earlier, we assign $\mathrm{k}$ with the value greater than 4 in order to discover the alpha helix containing one cycle at least. According to the fact that beta strand always connects another one laterally by three or more hydrogen bonds to forming pleated beta sheet, we assign $\mathrm{k}$ with the value greater than 3 for beta-strand finding.

Although the positions of all strands in primary structure (sequence) can be determined by formula(4), the count, position and orientation of beta sheets are still not clear. By considering the "sideways" distance between adjacent $\mathrm{C} \alpha$ atoms in hydrogen-bonded $\beta$ strands is roughly $5 \AA$, we build a band-pass filter with the range of [5$\left.\delta_{1}, 5+\delta_{2}\right]$ and perform it on distance matrix. As a result, whether $d m(i, i+k)$ is the indication of beta sheet or not can be determined by whether the following formula can be established or not

$$
-\delta_{1} \leq d m(i, i+k)-5 \leq \delta_{2} .
$$

\subsection{Feature Extraction of Protein Structure}

According to SCOP description, an all- $\alpha$ protein is a class of structural domains in which the secondary structure is composed entirely of $\alpha$-helices, with the possible exception of a few isolated $\beta$-sheets on the periphery. An all- $\beta$ protein is a class of structural domains in which the secondary structure is composed entirely of $\beta$-sheets, with the possible exception of a few isolated $\alpha$-helices on the periphery. An $\alpha / \beta$ protein is a class of structural domains which is composed of alternating $\alpha$-helices and mostly parallel $\beta$-strands along the backbone. An $\alpha+\beta$ proteins is a class of structural domains which is composed of $\alpha$-helices and mostly anti-parallel $\beta$-strands that occur 
separately along the backbone. If proteins belong to the same structural class, and have same major secondary structures in same arrangement and with the same topological connections, then they are grouped into a common fold.

Inspired by above facts, we regard composition of SSE as key feature in classification of structural class, and take arrangement of SSE, especially beta sheet, as important roles in classification of fold. First of all, we define the regions of interest (ROI) of distance matrix to extract feature for structural class and fold respectively. The first ROI looks like 5-element wide beam in distance matrix, while the second ROI is the little smaller triangle than the upper triangle part of distance matrix. Both of them are shown in Fig. 2 with different gray patterns.

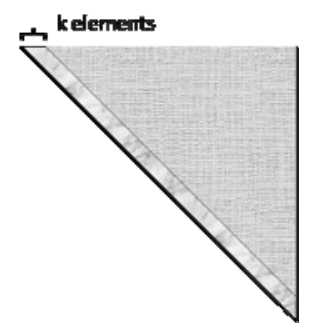

Fig. 2. Regions of Interest

The first feature of protein is called the composition of SSE and defined as

$$
F_{1}=\#\left\{S_{i}\right\} / N \text {, }
$$

where $\#\left\{S_{i}\right\}$ is the count of $S_{i}$ which satisfies formula (4).

In order to characterize the count, position and orientation of beta sheets, we split the second ROI into several smaller ones with multi-level decomposition and show them in Fig. 3.

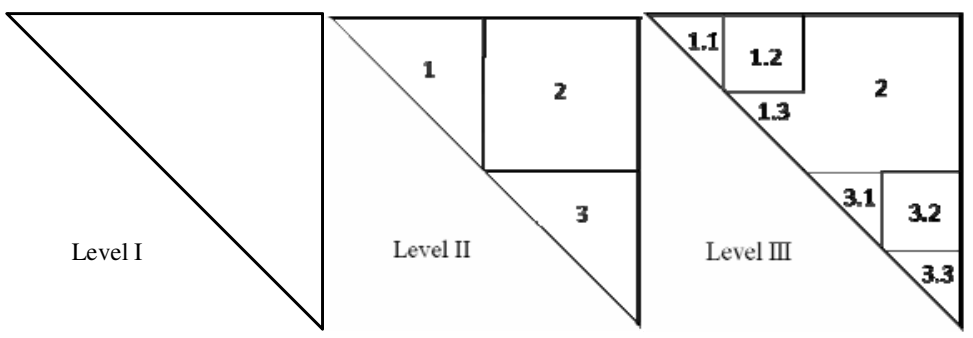

Fig. 3. Multi-level decomposed ROI with one or several sub-regions

As a result, the arrangement feature of protein is defined as

$$
F_{2}=\#\{d m(i, j)\} / C_{m, n},
$$




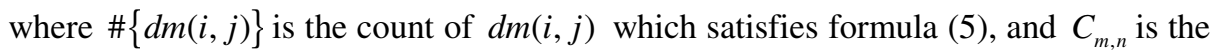
element count of the $\mathrm{n}^{\text {th }}$ sub-region in level $\mathrm{m}$.

Totally, $F_{1}$ is of two dimensions corresponding to alpha helix and beta strand, while the dimension of $F_{2}$ depends on the level of decomposition and equals $2^{m}-1$ where $m$ is the value of level.

\subsection{Classification}

Once the representation of protein structure is set, next step is to choose a classifier to perform classification. Support vector machines (SVM) has been used here due to its good performance of classification. SVM was originally designed for binary classification[25] while protein domain classification is of M-class classification. There are mainly two kinds of approaches for multi-class SVM[26]. The extensive experiments[10, 26] have shown that "One-Versus-Rest"(OVR), "One-Versus-One", (OVO) and "Directed Acyclic Graph" (DAG) are more practical. Because of its convenient usage, OVO is used in this paper. Practically, the SVM software, LibSVM, is used, and can be freely downloaded from http://www.csie.ntu.edu.tw/ cjlin/libsvm/ for academic research[26]. In addition, the training is done with only the $\mathrm{RBF}$ kernel in all experiments.

\section{Experiment}

\subsection{Classification Result of Structural Class and Fold}

Firstly, we use composition and arrangement features to perform classification of structural class and fold, respectively. In order to keep the dimension of feature vector as low as possible, only the decompositions of 3 levels are run when calculating arrangement feature. The results are listed in Table 1.

Table 1. The accuracy of classification with single group of features

\begin{tabular}{llll}
\hline Feature & Dimension & Structural class $(\%)$ & Fold(\%) \\
\hline $\mathrm{F}_{1}$ & 2 & 86.23 & 44.42 \\
$\mathrm{~F}_{2}$ Level 1 & 1 & 74.55 & 28.57 \\
$\mathrm{~F}_{2}$ Level 2 & 3 & 74.03 & 55.32 \\
$\mathrm{~F}_{2}$ Level 3 & 7 & 81.04 & 68.05 \\
\hline
\end{tabular}

Obviously, composition feature $\left(\mathrm{F}_{1}\right)$ is more effective to the classification of structural class, while arrangement feature $\left(\mathrm{F}_{2}\right)$ is more capable to characterize the divergence of different folds. In order to achieve a better classification, we combine composition feature spontaneously with arrangement feature and show the result in Table 2.

Table 2 shows that the combination of composition feature and arrangement feature of level 1 is enough to gain good result for structural class. The combination of composition feature and arrangement feature of level 3 wins the best result of fold classification. Besides, the greater the level of decomposition of the second ROI is, the higher the accuracy of fold classification is, but the higher the dimension of feature vector is. 
Table 2. The accuracy of classification with combined features

\begin{tabular}{llll}
\hline Feature & Dimension & Structural class $(\%)$ & Fold $(\%)$ \\
\hline $\mathrm{F}_{1}+\mathrm{F}_{2}$ Level 1 & 3 & 90.65 & 50.91 \\
$\mathrm{~F}_{1}+\mathrm{F}_{2}$ Level 2 & 5 & 91.17 & 64.42 \\
$\mathrm{~F}_{1}+\mathrm{F}_{2}$ Level 3 & 9 & 90.65 & 74.55 \\
\hline
\end{tabular}

Consequently, the decomposition level is determined by the requirement of running environment when building real application.

\subsection{Comparison with Former Methods}

In order to validate the effectiveness of the presented method, we compare it with several methods in different literatures of which all used the same benchmark dataset of original paper[5]. These methods can be categorized into two groups in the case of assessment approach. The first group [5, 7, 19, 20, 27]applies the training set to build classifier model and use independently the testing set to evaluate its performance. The second group[16,21] combines these two sets and assesses the performance of classification by 10 -cross validation. The comparison with the first group and the second group are listed in Table 3 and Table 4 respectively.

Table 3. The comparison in the independent test

\begin{tabular}{llll}
\hline Method & Dimension of feature & Structural class (\%) & Fold(\%) \\
\hline Ref. [5] & 20 & N/A & 49.4 \\
Ref. [5] & 125 & N/A & 56.5 \\
Ref. [27] & 125 & 80.52 & 58.18 \\
Ref. [19] & 125 & N/A & 61.04 \\
Ref. [20] & 1007 & 83.6 & 65.5 \\
Ref. [7] & 1007 & 87.0 & 69.6 \\
Our method & $\mathbf{3 / 9}$ & $\mathbf{9 0 . 6 5}$ & $\mathbf{7 4 . 5 5}$ \\
\hline
\end{tabular}

Table 4. The comparison in $10-\mathrm{CV}$ test

\begin{tabular}{llll}
\hline Method & Dimension of feature & Structural class (\%) & Fold $(\%)$ \\
\hline Ref. [21] & 125 & 84 & 74 \\
Ref. [16] & 183 & N/A & 78 \\
Our method & $3 / 9$ & $\mathbf{9 3 . 7 0}$ & $\mathbf{7 8 . 6 5}$ \\
\hline
\end{tabular}

The result demonstrates that our method outperforms extraordinarily other methods with both the highest accuracy of classification and the lowest dimension of feature vector. Besides, most of these methods exploited intricate techniques of pattern recognition, for example, feature fusion[19, 27], feature selection[7] and hierarchical classifier architecture[16, 20,21]. The techniques always increase the cost of building application. In contrast, the presented method is an agile solution of protein structure classification of SCOP. 


\section{Conclusion}

In this paper, we have developed a novel method of rapid domain classification. Instead of analyzing directly protein sequence or 3-D tertiary structure, the presented method maps firstly tertiary structure of protein domain into 2-D distance matrix. Then, two $\mathrm{C} \alpha-\mathrm{C} \alpha$ distance functions for alpha helix and beta strand are modeled by considering their geometrical properties respectively. After that, the distance functions are further applied to mine secondary structure elements in distance matrix with the way similar to image filtering. Furthermore, composition feature and arrangement feature of SSEs are presented to characterize domain structure for SCOP classification of structural class and fold. Finally, the results compared with other methods show that the presented method can perform effectively and efficiently automatic classification of domain with the benefit of low dimension and meaningful features, but also no need of complicated classifier system.

Acknowledgments. This work was supported by a grant from National Natural Science Foundation of China (60872145) and China Postdoctoral Science Foundation (20070421130).

\section{References}

1. Krissinel, E.: On the Relationship between Sequence and Structure Similarities in Proteomics. Bioinformatics 23, 717-723 (2007)

2. Andreeva, A., Howorth, D., Chandonia, J.-M., Brenner, S.E., Hubbard, T.J.P., Chothia, C., Murzin, A.G.: Data Growth and Its Impact on the SCOP Database: New Developments. Nucleic Acids Research 36, D419-D425 (2008)

3. Alison, L.C., Ian, S., Tony, L., Oliver, C.R., Richard, G., Janet, T., Christine, A.: The CATH Classification Revisited-Architectures Reviewed and New Ways to Characterize Structural Divergence in Superfamilies. Nucleic Acids Research 37, D310-D314 (2008)

4. Bastolla, U., Ortíz, A.R., Porto, M., Teichert, F.: Effective Connectivity Profile: A Structural Representation That Evidences the Relationship between Protein Structures and Sequences. Proteins: Structure, Function, and Bioinformatics 73, 872-888 (2008)

5. Ding, C.H.Q., Dubchak, I.: Multi-Class Protein Fold Recognition Using Support Vector Machines and Neural Networks. Bioinformatics 17, 349-358 (2001)

6. Shi, J.-Y., Zhang, S.-W., Liang, Y., Pan, Q.: Prediction of Protein Subcellular Localizations Using Moment Descriptors and Support Vector Machine. In: Rajapakse, J.C., Wong, L., Acharya, R. (eds.) PRIB 2006. LNCS (LNBI), vol. 4146, pp. 105-114. Springer, Heidelberg (2006)

7. Lin, K.L., Lin, C.-Y., Huang, C.-D., Chang, H.-M., Yang, C.-Y., Lin, C.-T., Tang, C.Y., Hsu, D.F.: Feature Selection and Combination Criteria for Improving Accuracy in Protein Structure Prediction. IEEE Transactions on NanoBioscience 6, 186-196 (2007)

8. Shi, J.-Y., Zhang, S.-W., Pan, Q., Zhou, G.-P.: Using Pseudo Amino Acid Composition to Predict Protein Subcellular Location: Approached with Amino Acid Composition Distribution. Amino Acids 35, 321-327 (2008)

9. Cai, Y.D., Liu, X.J., Xu, X.B., Chou, K.C.: Support Vector Machines for Prediction of Protein Subcellular Location by Incorporating Quasi-Sequence-Order Effect. Journal of Cellular Biochemistry 84, 343-348 (2002) 
10. Shi, J.-Y., Zhang, S.-W., Pan, Q., Cheng, Y.-M., Xie, J.: Prediction of Protein Subcellular Localization by Support Vector Machines Using Multi-Scale Energy and Pseudo Amino Acid Composition. Amino Acids 33, 69-74 (2007)

11. Ankerst, M., Kastenmüller, G., Kriegel, H.-P., Seidl, T.: 3D shape histograms for similarity search and classification in spatial databases. In: Güting, R.H., Papadias, D., Lochovsky, F.H. (eds.) SSD 1999. LNCS, vol. 1651, pp. 207-228. Springer, Heidelberg (1999)

12. Daras, P., Zarpalas, D., Axenopoulos, A., Tzovaras, D., Strintzis, M.G.: ThreeDimensional Shape-Structure Comparison Method for Protein Classification. IEEE Trans. Comput. Biol. Bioinformatics 3, 193-207 (2006)

13. Gilbert, D., Westhead, D., Viksna, J., Thornton, J.: A Computer System to Perform Structure Comparison Using Tops Representations of Protein Structure. Comput. Chem. 26, 23 $30(2001)$

14. Anne, P.: Voronoi and Voronoi-Related Tessellations in Studies of Protein Structure and Interaction. Current Opinion in Structural Biology 14, 233-241 (2004)

15. Choi, I.-G., Kwon, J., Kim, S.-H.: Local Feature Frequency Profile: A Method to Measure Structural Similarity in Proteins. Proceedings of the National Academy of Sciences of the United States of America 101, 3797-3802 (2004)

16. Marsolo, K., Parthasarathy, S.: Alternate Representation of Distance Matrices for Characterization of Protein Structure. In: Proceedings of the Fifth IEEE International Conference on Data Mining, pp. 298-305. IEEE Computer Society, Los Alamitos (2005)

17. Sayre, T., Singh, R.: Protein Structure Comparison and Alignment Using Residue Contexts. In: Proceedings of the 22nd International Conference on Advanced Information Networking and Applications - Workshops, pp. 796-801. IEEE Computer Society, Los Alamitos (2008)

18. Shi, J.-Y., Zhang, S.-W., Pan, Q., Zhang, Y.-N.: Using Decision Templates to Predict Subcellular Localization of Protein. In: Rajapakse, J.C., Schmidt, B., Volkert, L.G. (eds.) PRIB 2007. LNCS (LNBI), vol. 4774, pp. 71-83. Springer, Heidelberg (2007)

19. Shi, J.-Y., Zhang, S.-W., Pan, Q., Liang, Y.: Protein Fold Recognition with Support Vector Machines Fusion Network. Progress in Biochemistry and Biophysics 33, 155-162 (2006)

20. Huang, C.-D., Lin, C.-T., Pal, N.R.: Hierarchical Learning Architecture with Automatic Feature Selection for Multiclass Protein Fold Classification. IEEE Transactions on NanoBioscience 2, 221-232 (2003)

21. Marsolo, K., Parthasarathy, S., Ding, C.: A Multi-Level Approach to SCOP Fold Recognition. In: Proceedings of the Fifth IEEE Symposium on Bioinformatics and Bioengineering, pp. 57-64. IEEE Computer Society, Los Alamitos (2005)

22. Chandonia, J., Hon, G., Walker, N., Lo Conte, L., Koehl, P., Levitt, M., Brenner, S.: The Astral Compendium in 2004. Nucleic Acids Research 32, D189-D192 (2004)

23. Taylor, W.R., Orengo, C.A.: Protein Structure Alignment. J. Mol. Biol. 208, 1-22 (1989)

24. Timothy, H., Irwin, K., Gordon, C.: The Theory and Practice of Distance Geometry. Bulletin of Mathematical Biology 45, 665-720 (1983)

25. Vapnik, V.N.: An Overview of Statistical Learning Theory. IEEE Transactions on Neural Networks 10, 988-999 (1999)

26. Hsu, C., Lin, C.J.: A Comparison of Methods for Multi-Class Support Vector Machines. IEEE Transactions on Neural Networks 13, 415-425 (2002)

27. Chinnasamy, A., Sung, W.K., Mittal, A.: Protein Structure and Fold Prediction Using Tree-Augmented Naive Bayesian Classifier. Journal of Bioinformatics and Computational Biology 3, 803-820 (2005) 